\title{
Application of Piecewise Successive Linearization Method for the Solutions of the Chen Chaotic System
}

\author{
S. S. Motsa, ${ }^{1}$ Y. Khan, ${ }^{2}$ and S. Shateyi ${ }^{3}$ \\ ${ }^{1}$ School of Mathematical Sciences, University of KwaZulu-Natal, Private Bag X01, Pietermaritzburg, \\ Scottsville 3209, South Africa \\ ${ }^{2}$ Department of Mathematics, Zhejiang University, Hangzhou 310027, China \\ ${ }^{3}$ Department of Mathematics, University of Venda, Private Bag X5050, Thohoyandou 0950, South Africa
}

Correspondence should be addressed to S. Shateyi, stanford.shateyi@univen.ac.za

Received 18 April 2012; Revised 17 June 2012; Accepted 18 June 2012

Academic Editor: Hak-Keung Lam

Copyright (C) 2012 S. S. Motsa et al. This is an open access article distributed under the Creative Commons Attribution License, which permits unrestricted use, distribution, and reproduction in any medium, provided the original work is properly cited.

This paper centres on the application of the new piecewise successive linearization method (PSLM) in solving the chaotic and nonchaotic Chen system. Numerical simulations are presented graphically and comparison is made between the PSLM and Runge-Kutta-based methods. The work shows that the proposed method provides good accuracy and can be easily extended to other dynamical systems including those that are chaotic in nature.

\section{Introduction}

In this paper, we consider the Chen dynamical system [1], which is a three-dimensional system of ordinary differential equations with quadratic nonlinearities, defined as

$$
\begin{gathered}
\frac{d x}{d t}=a(y-x), \\
\frac{d y}{d t}=(c-a) x-x z+c y, \\
\frac{d z}{d t}=x y-b z,
\end{gathered}
$$


where $x, y, z$ are dependent variables and $a, b, c$ are positive constants. The Chen system (1.1) does not admit a closed form solution and it is known to exhibit both chaotic and nonchaotic behaviors for distinct parameter values. Bifurcation studies [2-4] have shown that with the parameters $a=35$ and $c=28$, system (1.1) exhibits nonchaotic behavior and chaotic behavior when $b=12$ and $b=3$, respectively. Finding accurate and efficient methods for solving chaotic systems such as the Chen system has been an active research area for many researchers who use such systems as a benchmark for testing the accuracy and performance of methods of solutions under their development. Other variants of the Chen system such as fractional order and four-dimensional Chen systems have been the subject of study by many scholars.

The solution of nonchaotic, chaotic and hyperchaotic Chen system equations has been obtained by different analytical and numerical methods such as Runge-Kutta-based numerical schemes. In recent years, several analytical and seminumerical methods have been utilized in solving the Chen system equations $[5,6]$. Because the solutions for the Chen systems rapidly oscillate within small intervals, conventional analytical and numerical algorithms have been implemented in a sequence of small multiple intervals in order to ensure accuracy convergence of the solution techniques. Recent multistage methods that have been reported for solving Chen systems include the multistage differential transform method $[7,8]$, the multistage homotopy analysis method [9], the multistep homotopy perturbation method [10] and the multistage variational iteration method [11].

The aim of this paper is to obtain the solution of the Chen system using the successive linearization method (SLM) that is implemented in a sequence of intervals. This new algorithm is called the piecewise successive linearization method (PSLM). The standard SLM was developed primarily for solving nonlinear boundary value problems (see, e.g., [12-17]). To the best of our knowledge, the method has not been extended to initial value problem (IVPs) and in particular chaotic IVPs of the type (1.1). We remark that the standard SLM is not suitable for solving dynamical systems of the type (1.1), hence the need to modify the method and implement it on multiple intervals. The paper focuses on the use of the proposed PSLM in solving the Chen system (1.1) using selected parameters that yield both chaotic and nonchaotic results. Numerical results are presented graphically and are compared with Runge-Kutta-based results and excellent agreement is observed.

The remainder of the paper is organized as follows. In Section 2, the basic description of the standard SLM is briefly presented. In Section 3, the PSLM is introduced. The application of the proposed method is numerically investigated in Section 4 to generate results which are discussed therein. Finally, conclusions in Section 5 close the paper.

\section{Successive Linearization Method (SLM)}

In this section, we give a brief description of the basic idea behind the successive linearization method (SLM) [13-15]. This will be followed by a description of the multistage extension of the SLM algorithm which is applied to the Chen system (1.1).

The SLM approach assumes that the solution of (1.1) can be expressed as

$$
x(t)=x_{s}(t)+\sum_{m=0}^{s-1} x_{m}(t), \quad y(t)=y_{s}(t)+\sum_{m=0}^{s-1} y_{m}(t), \quad z(t)=z_{s}(t)+\sum_{m=0}^{s-1} z_{m}(t) .
$$


Substituting (2.1) in the governing equation (1.1) and neglecting nonlinear terms in $x_{s}, y_{s}, z_{s}$ we obtain

$$
\begin{gathered}
\frac{d x_{s}}{d t}+a x_{s}-a y_{s}=r_{1, s-1}, \\
\frac{d y_{s}}{d t}-c y_{s}+\alpha_{1, s-1} x_{s}+\alpha_{2, s-1} z_{s}=r_{2, s-1} \\
\frac{d z_{s}}{d t}+b z_{s}-\beta_{1, s-1} x_{s}-\beta_{2, s-1} y_{s}=r_{3, s-1},
\end{gathered}
$$

subject to the initial conditions

$$
x_{s}(0)=y_{s}(0)=z_{s}(0)=0,
$$

where

$$
\begin{gathered}
\alpha_{1, s-1}=a-c+\sum_{m=0}^{s-1} z_{m}, \quad \alpha_{2, s-1}=\sum_{m=0}^{s-1} x_{m}, \\
\beta_{1, s-1}=\sum_{m=0}^{s-1} y_{m}, \quad \beta_{2, s-1}=\sum_{m=0}^{s-1} x_{m}, \\
r_{1, s-1}=-\sum_{m=0}^{s-1}\left(\frac{d x_{m}}{d t}-a y_{m}+a x_{m}\right), \\
r_{2, s-1}=-\sum_{m=0}^{s-1}\left(\frac{d y_{m}}{d t}-(c-a) x_{m}-c y_{m}\right)+\left(\sum_{m=0}^{s-1} x_{m}\right)\left(\sum_{m=0}^{s-1} z_{m}\right), \\
r_{3, s-1}=-\sum_{m=0}^{s-1}\left(\frac{d z_{m}}{d t}+b z_{m}\right)+\left(\sum_{m=0}^{s-1} x_{m}\right)\left(\sum_{m=0}^{s-1} y_{m}\right) .
\end{gathered}
$$

Starting from a suitable initial approximation $x_{0}(t), y_{0}(t), z_{0}(t)$, the solutions for $x_{s}(t), y_{s}(t)$, $z_{s}(t)$ (for $s=1,2,3, \ldots$ ) can be obtained by iteratively solving the linear system (2.2)-(2.4). A suitable initial guess to start off the SLM algorithm (2.2)-(2.4) is obtained by solving the linear part of (1.1) subject to the problem's initial condition $x(0)=x_{0}, y(0)=y_{0}, z(0)=z_{0}$ where $x_{0}, y_{0}, z_{0}$ are given initial values. For this system of first-order equations the initial approximation can easily be solved analytically in any given interval $t \in\left[t_{0}, t_{n}\right]$. However, the solution of the SLM algorithm (2.2)-(2.4) cannot be solved exactly. Spectral collocation methods (or any other numerical method) can be used to solve (2.2)-(2.4) in any given interval $t \in\left[t_{0}, t_{n}\right]$. After obtaining solutions for (2.3), the approximate solution $x(t), y(t)$, and $z(t)$ is determined as the series solution

$$
x(t)=x_{0}(t)+x_{1}(t)+x_{2}(t)+\cdots .
$$


Similar expressions are obtained for $y(t)$ and $z(t)$. A SLM solution is said to be of order $M$ if the above series is truncated at $s=M$, that is, if

$$
x(t)=\sum_{m=0}^{M} x_{m}(t) .
$$

\section{Piecewise Successive Linearization Method}

It was observed through numerical experimentation that, in its standard form, the SLM will not give accurate solutions for initial value problems of the Chen system type. Thus, it can be remarked that the SLM is ideally suited for boundary value problems whose solutions do not rapidly change in behaviour or oscillate over small regions of the domain of the governing problem. The SLM solution can thus be considered to be local in nature and may not be suitable for initial value problems at very large values of the independent variable $t$. A simple way of ensuring the validity of the approximations for all values of $t$ is to determine the solution in a sequence of equal intervals, which are subject to continuity conditions at the end points of each interval. To extend this solution over the interval $\Omega=\left[t^{0}, t^{n}\right]$, we divide the interval $\Omega$ into sub-intervals $\Omega_{i}=\left[t^{i-1}, t^{i}\right], i=1,2,3, \ldots, n$ where $t^{0} \leq t^{1} \leq \cdots \leq t^{n}$. We solve (2.2)-(2.4) in each subinterval $\Omega_{i}$. When applied to the multiple subintervals the SLM will be referred to as the piecewise SLM (PSLM).

Let $x^{1}(t), y^{1}(t), z^{1}(t)$ be the solution of (2.2)-(2.4) in the first subinterval $\left[t^{0}, t^{1}\right]$ and $x^{i}(t), y^{i}(t), z^{i}(t)$ be the solutions in the subintervals $\Omega_{i}$ for $2 \leq i \leq n$. The initial conditions used in obtaining the solutions in the subinterval $\Omega_{i}(2 \leq i \leq n)$ are obtained from the initial conditions of the subinterval $\Omega_{i-1}$. Thus, we solve

$$
\begin{gathered}
\frac{d x_{s}^{i}}{d t}+a x_{s}^{i}-a y_{s}^{i}=r_{1, i-1}^{i}, \\
\frac{d y_{s}^{i}}{d t}-c y_{s}^{i}+\alpha_{1, s-1}^{i} x_{s}^{i}+\alpha_{2, s-1}^{i} z_{s}^{i}=r_{2, s-1}^{i}, \\
\frac{d z_{s}^{i}}{d t}+b z_{s}^{i}-\beta_{1, s-1}^{i} x_{s}^{i}-\beta_{2, s-1}^{i} y_{s}^{i}=r_{3, s-1}^{i},
\end{gathered}
$$

subject to the initial conditions

$$
x_{s}^{i}\left(t^{i-1}\right)=y_{s}^{i}\left(t^{i-1}\right)=z_{s}^{i}\left(t^{i-1}\right)=0
$$

The initial approximations for solving (3.1) are obtained as solutions of the system

$$
\begin{gathered}
\frac{d x_{0}^{i}}{d t}=a\left(y_{0}^{i}-x_{0}^{i}\right) \\
\frac{d y_{0}^{i}}{d t}=(c-a) x_{0}^{i}+c y_{0}^{i} \\
\frac{d z_{0}^{i}}{d t}=-b z_{0}^{i}
\end{gathered}
$$


subject to the initial condition

$$
x_{0}^{i}\left(t^{i-1}\right)=x^{i-1}\left(t^{i-1}\right), \quad y_{0}^{i}\left(t^{i-1}\right)=y^{i-1}\left(t^{i-1}\right), \quad z_{0}^{i}\left(t^{i-1}\right)=z^{i-1}\left(t^{i-1}\right) .
$$

An exact solution of (3.3) can easily be found. To solve (3.1) we use the Chebyshev spectral collocation method on each interval $\left[t^{i-1}, t^{i}\right]$. Before applying the spectral method the region $\left[t^{i-1}, t^{i}\right]$ is transformed to the interval $[-1,1]$ on which the spectral collocation method is defined. This can be achieved by using the linear transformation

$$
t=\frac{\left(t^{i}-t^{i-1}\right) \tau}{2}+\frac{\left(t^{i}+t^{i-1}\right)}{2}, \quad \tau \in[-1,1]
$$

in each interval $\left[t^{i-1}, t^{i}\right]$ (for $i=1, \ldots, n$ ), After the transformation, the interval $\left[t^{i-1}, t^{i}\right]$ is discretized using the Chebyshev-Gauss-Lobatto collocation points [18, 19]

$$
\tau_{j}^{i}=\cos \left(\frac{\pi j}{N}\right), \quad j=0,1, \ldots, N
$$

which are the extrema of the $N$ th-order Chebyshev polynomial

$$
T_{N}(\tau)=\cos \left(N \cos ^{-1} \tau\right)
$$

The basic idea behind the pseudo-spectral collocation method is the introduction of a differentiation matrix $D$ which is used to approximate the derivatives of the unknown variables $x_{s}^{i}(t), y_{s}^{i}(t), z_{s}^{i}(t)$ at the collocation points as the matrix vector product

$$
\frac{d x_{s}^{i}}{d t}=\sum_{k=0}^{N} \mathbf{D}_{j k} x_{s}^{i}\left(\tau_{k}^{i}\right)=\mathbf{D X}_{s}^{i}, \quad j=0,1, \ldots, N
$$

where $\mathbf{D}=2 D /\left(t^{i}-t^{i-1}\right)$ and $\mathbf{X}_{s}^{i}=\left[x_{s}^{i}\left(\tau_{0}^{i}\right), x_{s}^{i}\left(\tau_{2}^{i}\right), \ldots, x_{s}^{i}\left(\tau_{N}^{i}\right)\right]^{T}$ is the vector function at the collocation points $\tau_{j}^{i}$. Similar expressions can be obtained for $y_{s}^{i}(t)$ and $z_{s}^{i}(t)$. The Chebyshev derivative matrix $D$ is of size $(N+1) \times(N+1)$ and its entries are defined $[18,19]$ as

$$
\begin{gathered}
D_{j k}=\frac{c_{j}}{c_{k}} \frac{(-1)^{j+k}}{z_{j}-z_{k}}, \quad j \neq k ; j, k=0,1, \ldots, N, \\
D_{k k}=-\frac{z_{k}}{2\left(1-z_{k}^{2}\right)}, \quad k=1,2, \ldots, N-1, \\
D_{00}=\frac{2 N^{2}+1}{6}=-D_{N N},
\end{gathered}
$$


with

$$
c_{k}= \begin{cases}2 & k=0, N \\ 1 & -1 \leq k \leq N-1\end{cases}
$$

Applying the Chebyshev spectral collocation method in (3.1) gives

$$
\mathbf{A}_{s-1}^{i} \mathbf{F}_{s}^{i}=\mathbf{R}_{s-1}^{i}, \quad \mathbf{F}_{s}^{i}\left(\tau_{N}^{i-1}\right)=0,
$$

where

$$
\mathbf{A}_{s-1}^{i}=\left[\begin{array}{ccc}
\mathbf{D}+a \mathbf{I} & -a \mathbf{I} & \mathbf{O} \\
\widetilde{\alpha}_{1, s-1}^{i} & \mathbf{D}-c \mathbf{I} & \widetilde{\alpha}_{2, s-1}^{i} \\
-\widetilde{\beta}_{1, s-1}^{i} & -\widetilde{\beta}_{2, s-1}^{i} & \mathbf{D}+b \mathbf{I}
\end{array}\right], \quad \mathbf{R}_{s-1}^{i}=\left[\begin{array}{c}
\mathbf{r}_{1, s-1}^{i} \\
\mathbf{r}_{2, s-1}^{i} \\
\mathbf{r}_{3, s-1}^{i}
\end{array}\right], \quad \mathbf{F}_{s}^{i}=\left[\begin{array}{c}
\mathbf{X}_{s}^{i} \\
\mathbf{Y}_{s}^{i} \\
\mathbf{Z}_{s}^{i}
\end{array}\right]
$$

and $\mathbf{I}$ is an $(N+1) \times(N+1)$ identity matrix, $\mathbf{O}$ is an $(N+1) \times(N+1)$ matrix of zeros. The $\tilde{()}$ denotes an $(N+1) \times(N+1)$ diagonal matrix corresponding to () that is evaluated at the collocation points and $\mathbf{r}_{k, s-1}^{i}(k=1,2,3)$ is an $(N+1) \times 1$ vector corresponding to the quantities $r_{k, s-1}^{i}(t)$ that have been evaluated at the collocation points. Thus, starting from the initial approximation obtained as a solution of (3.3)-(3.4), the recurrence formula

$$
\mathbf{F}_{s}^{i}=\left(\mathbf{A}_{s-1}^{i}\right)^{-1} \mathbf{R}_{s-1}^{i}
$$

can be used to obtain the solutions $x^{i}(t), y^{i}(t), z^{i}(t)$ in the interval $\left[t^{i}, t^{i-1}\right]$. The solution approximating $x(t)$, for example, in the entire interval $\left[t^{0}, t^{n}\right]$ is given by

$$
x(t)= \begin{cases}x^{1}(t), & t \in\left[t^{0}, t^{1}\right] \\ x^{2}(t), & t \in\left[t^{1}, t^{2}\right] \\ \vdots & \\ x^{n}(t), & t \in\left[t^{n-1}, t^{n}\right] .\end{cases}
$$

Similar expressions can be obtained for $y(t)$ and $z(t)$. We observe that when $n=1$, the proposed piecewise successive linearization method (PSLM) becomes equivalent to the original SLM algorithm.

\section{Results and Discussion}

In this section, numerical simulations are given to validate the proposed piecewise successive linearization method. The PSLM results were obtained using $N=10$ collocation points and ten iterations (that is $M=10$ ) in each $\left[t^{i-1}, t^{i}\right]$ interval. Unless otherwise specified, the width of each interval $\Delta t=t^{i}-t^{i-1}$ was taken to be $\Delta t=0.1$. We also fix the values of the parameters $a=35$ and $c=28$ with $b=12$ for the nonchaotic case and $b=3$ for the chaotic case. The initial 


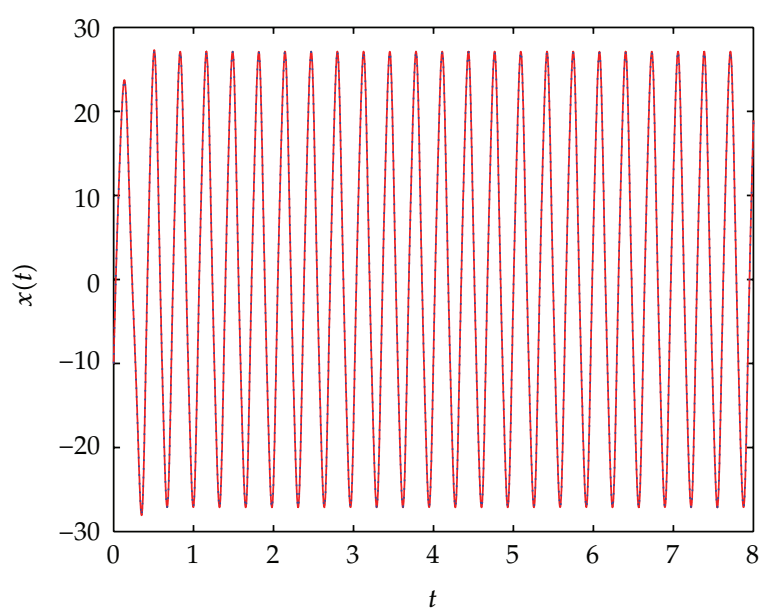

(a)

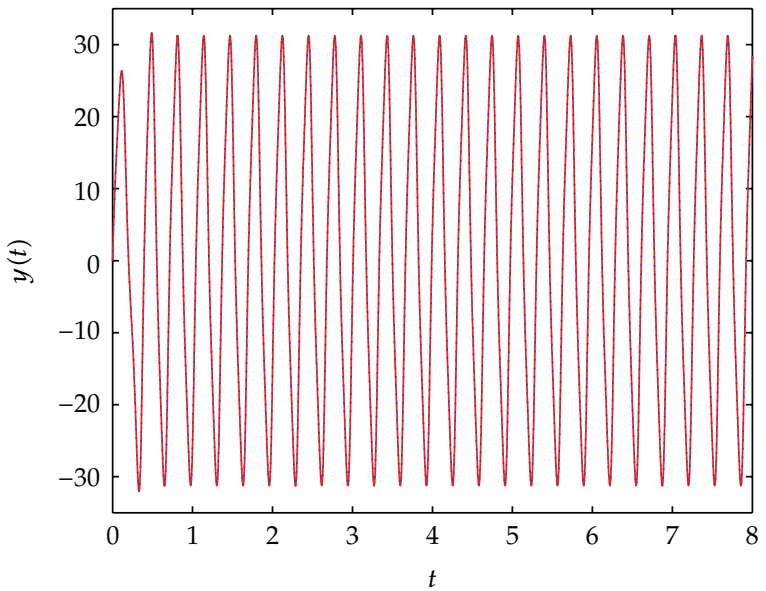

(b)

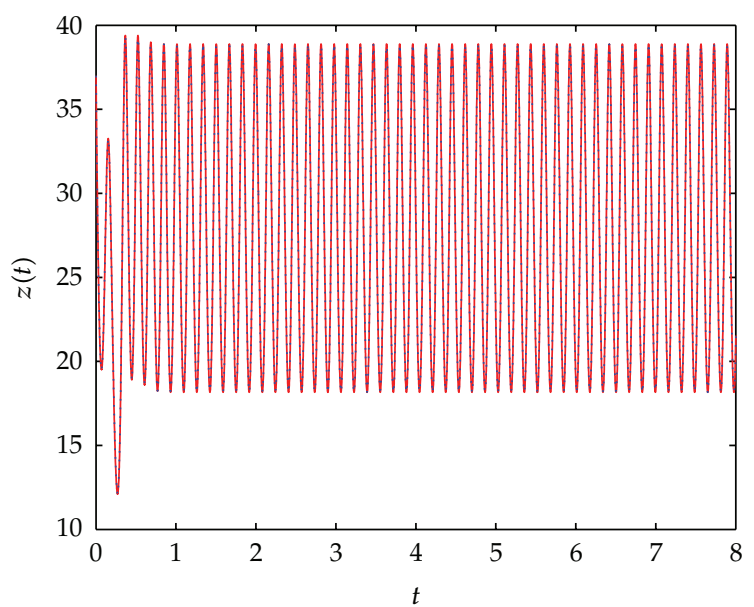

(c)

Figure 1: Time series PSLM (solid line) and ode45 (dots) results for nonchaotic case with parameters $a=35, b=12$, and $c=28$. 


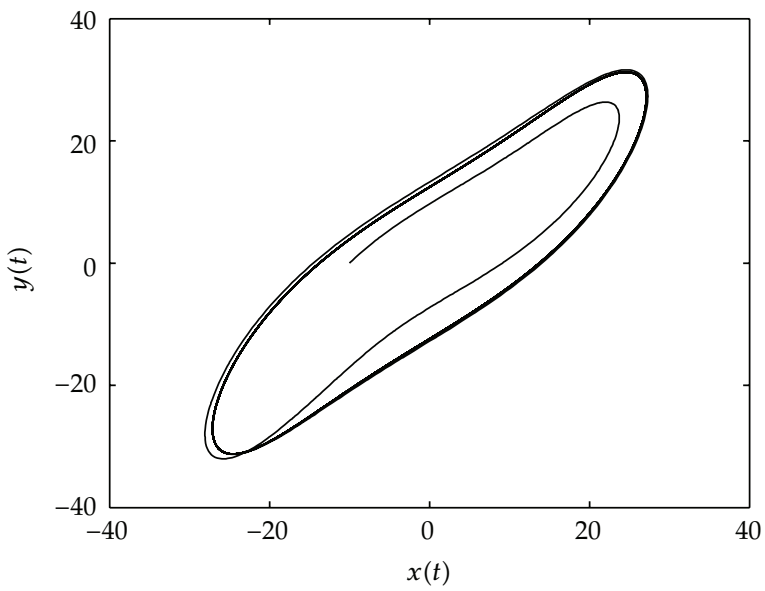

(a)

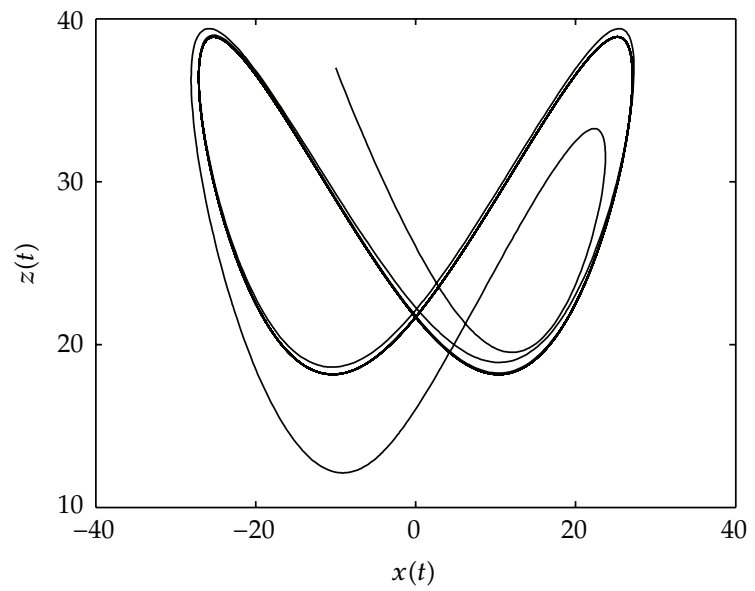

(b)

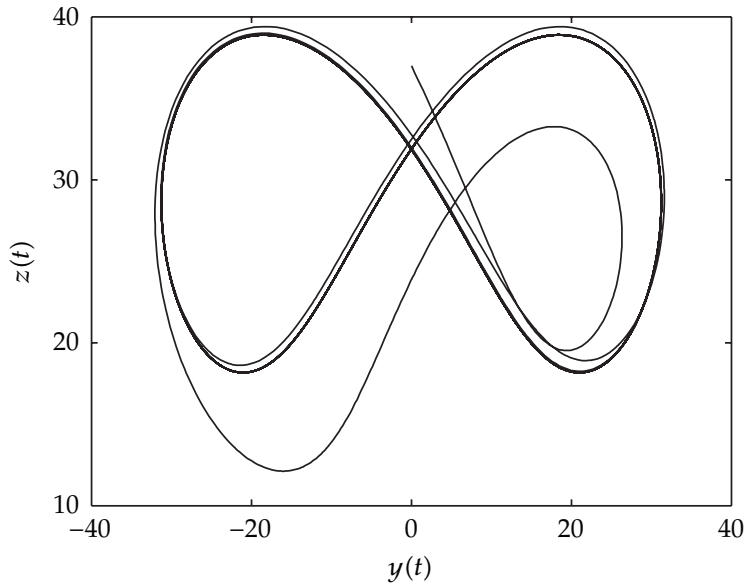

(c)

Figure 2: Phase portraits of the Chen system using the PSLM for the nonchaotic case. 


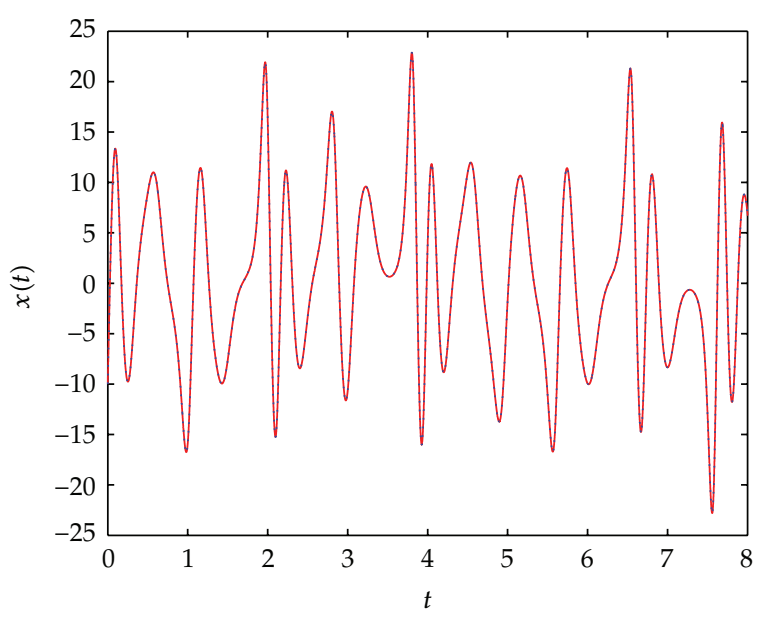

(a)

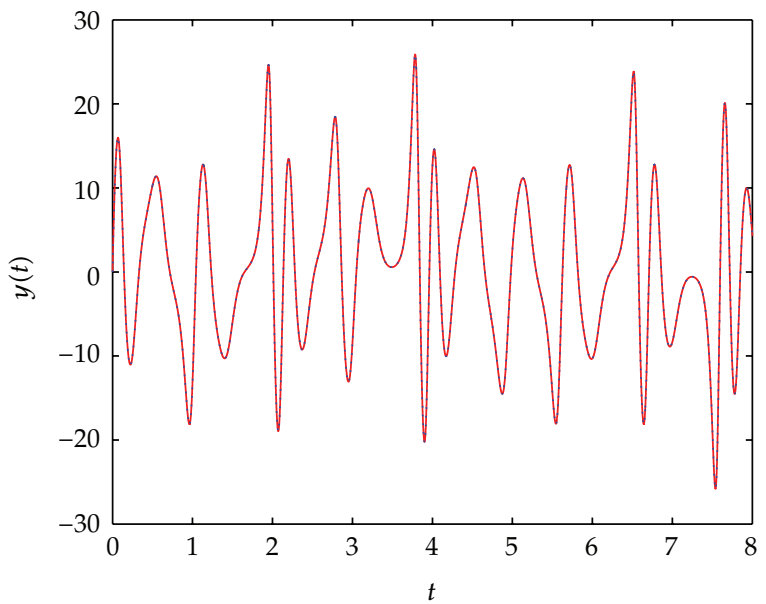

(b)

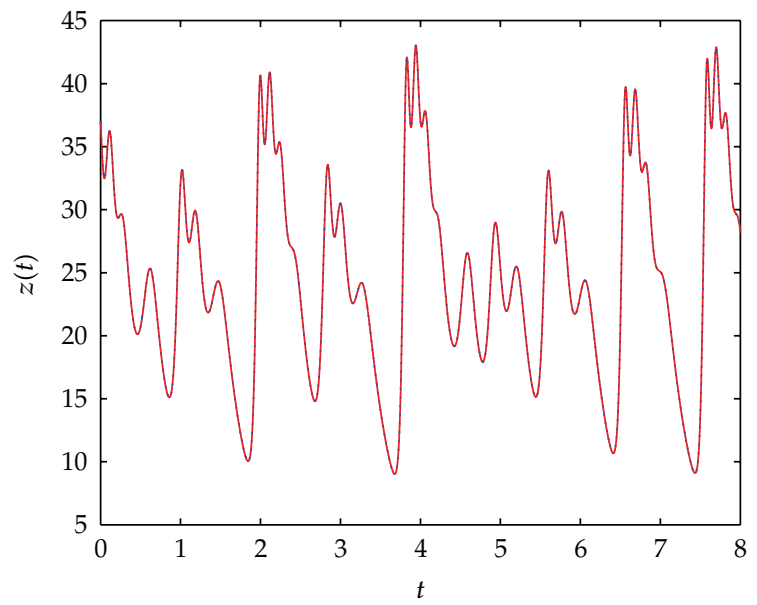

(c)

Figure 3: Time series PSLM (solid line) and ode45 (dots) results for nonchaotic case with parameters $a=35, b=3$, and $c=28$. 


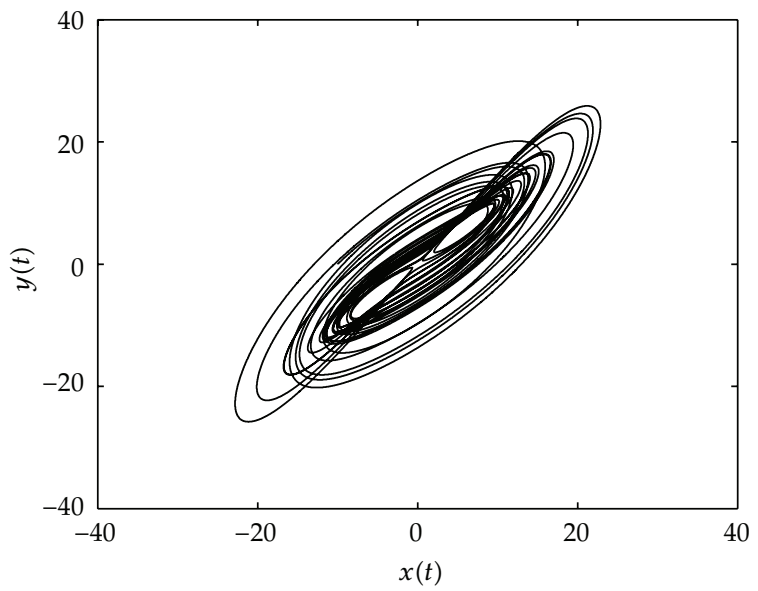

(a)

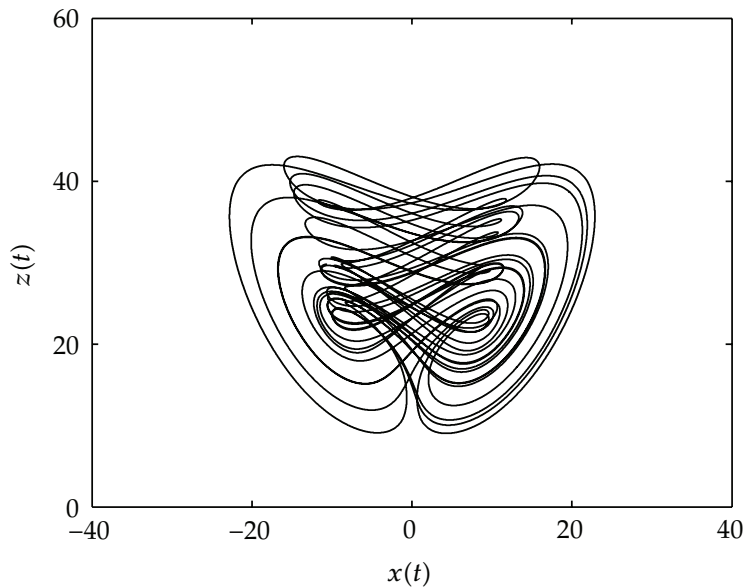

(b)

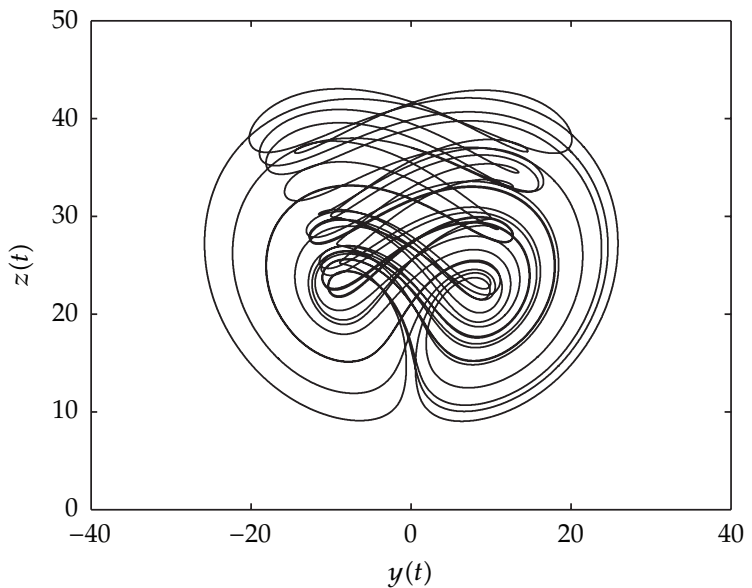

(c)

Figure 4: Phase portraits of the Chen system using the PSLM for the chaotic case. 
conditions are $x(0)=-10, y(0)=0$, and $z(0)=37$. The results for all the simulations done in this paper are computed for the time span $t \in[0,10]$. In order to assess the accuracy and performance of the proposed PSLM approach, the present results are compared with those obtained with the MATLAB built-in solver ode 45 . The ode 45 solver integrates a system of ordinary differential equations using explicit 4th and 5th Runge-Kutta $(4,5)$ formula, the Dormand-Prince pair [20].

The results of the PSLM simulation of the Chen system for the nonchaotic case are shown in Figures 1 and 2. In Figure 1 the results of the comparison between the PSLM and Runge-Kutta results for the time series evolution of the state variables are given. It can be seen that there is good agreement between the two results. Figure 2 depicts the phase portraits for the nonchaotic case. We observe that the system converges to a periodic orbit and results in a limit cycle as shown in Figure 2 when the parameters $a=35, b=12$, and $c=28$ are used.

The results of the PSLM simulation of the Chen system for the chaotic case are shown in Figures 3 and 4. In Figure 3, the PSLM results are compared with the Runge-Kutta results when parameters $a=35, c=28$, and $b=3$ are used. For this set of parameters, the system becomes chaotic and the chaotic attractors are shown in Figure 4. We observe that there is good agreement between the PSLM and Runge-Kutta results in the time series illustration depicted in Figure 3. This shows that the proposed method of solution has great potential of being a useful tool for solving complex dynamical systems with chaotic and nonchaotic behaviour.

\section{Conclusion}

In this paper, we carefully develop a novel piecewise successive linearization method (PSLM) which seeks to overcome the difficulties arising when the successive linearization method is extended to initial value problems with complex behaviour. The proposed method of solution is implemented on the Chen system and results are generated using known parameters that give both chaotic and nonchaotic outcomes. The validity of PSLM is verified by comparing its results with the results of built in Matlab-based numerical Runge-Kutta method, ode45. Close agreement of the two sets of results is observed thus demonstrating the accuracy of the PSLM approach for the particular problem considered. In conclusion, the PSLM may be considered as a robust refinement and extension of the existing SLM. The extended method may also be valid for other complex nonlinear initial value problems with chaotic behaviour. This paper can be used as a standard example for other applications in engineering and applied sciences.

\section{References}

[1] G. Chen and T. Ueta, "Yet another chaotic attractor," International Journal of Bifurcation and Chaos in Applied Sciences and Engineering, vol. 9, no. 7, pp. 1465-1466, 1999.

[2] C. P. Li and G. R. Chen, "A note one Hopf bifurcation in Chen's system," International Journal of Bifurcation and Chaos, vol. 13, no. 6, pp. 1609-1615, 2003.

[3] J. Lü, T. Zhou, G. Chen, and S. Zhang, "Local bifurcations of the Chen system," International Journal of Bifurcation and Chaos, vol. 12, no. 10, pp. 2257-2270, 2002.

[4] T. Ueta and G. Chen, "Bifurcation analysis of Chen's equation," International Journal of Bifurcation and Chaos, vol. 10, no. 8, pp. 1917-1931, 2000.

[5] A. K. Alomari, M. S. M. Noorani, and R. Nazar, "Homotopy approach for the hyperchaotic Chen system," Physica Scripta, vol. 81, Article ID 045005, 2010.

[6] M. S. M. Noorani, I. Hashim, R. Ahmad, S. A. Bakar, E. S. Ismail, and A. M. Zakaria, "Comparing numerical methods for the solutions of the Chen system," Chaos, Solitons E Fractals, vol. 32, p. 1296, 2007. 
[7] A. K. Alomari, "A new analytic solution for fractional chaotic dynamical systems using the differential transform method," Computers $\mathcal{E}$ Mathematics with Applications, vol. 61, no. 9, pp. 25282534, 2011.

[8] Z. M. Odibat, C. Bertelle, M. A. Aziz-Alaoui, and G. H. E. Duchamp, "A multi-step differential transform method and application to non-chaotic or chaotic systems," Computers $\mathcal{E}$ Mathematics with Applications, vol. 59, no. 4, pp. 1462-1472, 2010.

[9] A. K. Alomari, M. S. M. Noorani, and R. Nazar, "Adaptation of homotopy analysis method for the numericanalytic solution of Chen system," Communications in Nonlinear Science and Numerical Simulation, vol. 14, p. 2336, 2009.

[10] M. S. H. Chowdhury and I. Hashim, "Application of multistage homotopy-perturbation method for the solutions of the Chen system," Nonlinear Analysis, vol. 10, no. 1, pp. 381-391, 2009.

[11] S. M. Goh, M. S. M. Noorani, and I. Hashim, "On solving the chaotic Chen system: a new time marching design for the variational iteration method using Adomian's polynomial," Numerical Algorithms, vol. 54, no. 2, pp. 245-260, 2010.

[12] F. G. Awad, P. Sibanda, S. S. Motsa, and O. D. Makinde, "Convection from an inverted cone in a porous medium with cross-diffusion effects," Computers $\mathcal{E}$ Mathematics with Applications, vol. 61, no. 5, pp. 1431-1441, 2011.

[13] Z. G. Makukula, P. Sibanda, and S. S. Motsa, "A note on the solution of the von Kármán equations using series and Chebyshev spectral methods," Boundary Value Problems, vol. 2010, Article ID 471793, 17 pages, 2010.

[14] S. S. Motsa, "New algorithm for solving non-linear BVPs in heat transfer," International Journal of Modeling, Simulation E Scientific Computing, vol. 2, no. 3, pp. 355-373, 2011.

[15] S. S. Motsa, P. Sibanda, and S. Shateyi, "On a new quasi-linearization method for systems of nonlinear boundary value problems," Mathematical Methods in the Applied Sciences, vol. 34, no. 11, pp. 1406-1413, 2011.

[16] S. S. Motsa and P. Sibanda, "A linearisation method for non-linear singular boundary value problems," Computers \& Mathematics with Applications, vol. 63, no. 7, pp. 1197-1203, 2012.

[17] S. Shateyi and S. S. Motsa, "Variable viscosity on magnetohydrodynamic fluid flow and heat transfer over an unsteady stretching surface with Hall effect," Boundary Value Problems, vol. 2010, Article ID 257568, 20 pages, 2010.

[18] C. Canuto, M. Y. Hussaini, A. Quarteroni, and T. A. Zang, Spectral Methods in Fluid Dynamics, Springer Series in Computational Physics, Springer, Berlin, Germany, 1988.

[19] L. N. Trefethen, Spectral Methods in MATLAB, vol. 10 of Software, Environments, and Tools, Society for Industrial and Applied Mathematics (SIAM), Philadelphia, Pa, USA, 2000.

[20] J. R. Dormand and P. J. Prince, "A family of embedded Runge-Kutta formulae," Journal of Computational and Applied Mathematics, vol. 6, no. 1, pp. 19-26, 1980. 


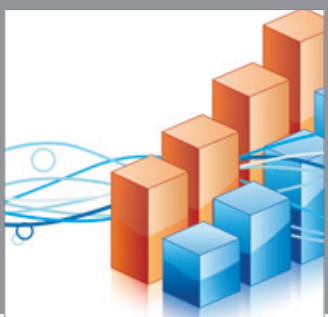

Advances in

Operations Research

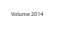

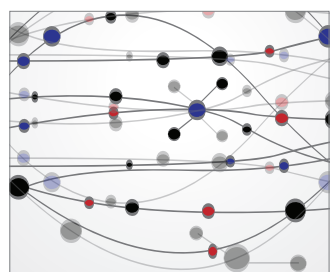

\section{The Scientific} World Journal
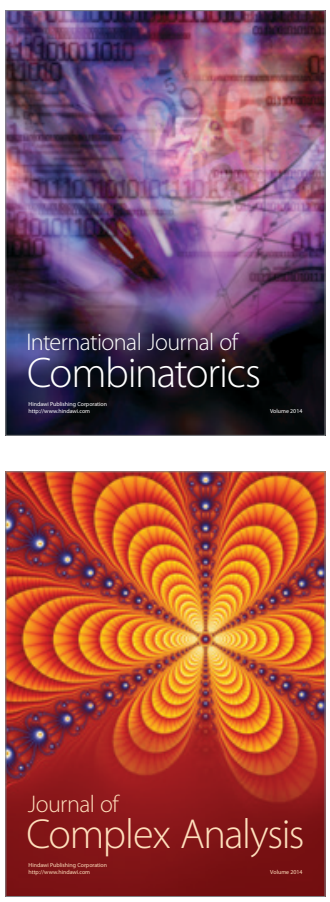

International Journal of

Mathematics and

Mathematical

Sciences
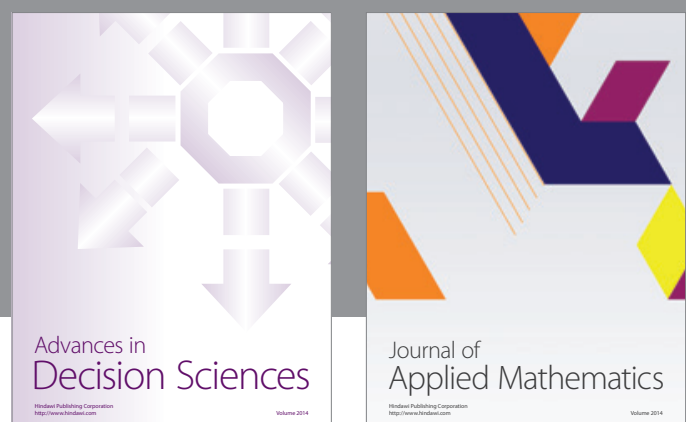

Journal of

Applied Mathematics
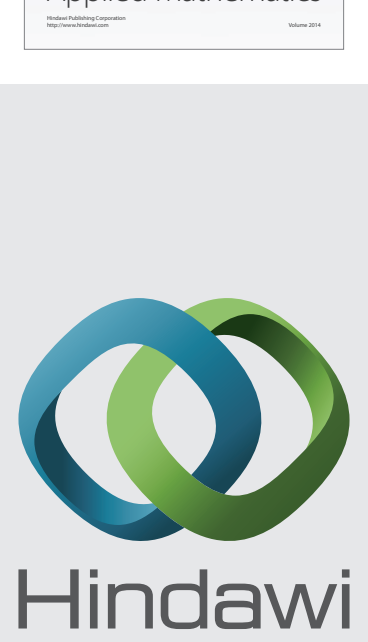

Submit your manuscripts at http://www.hindawi.com
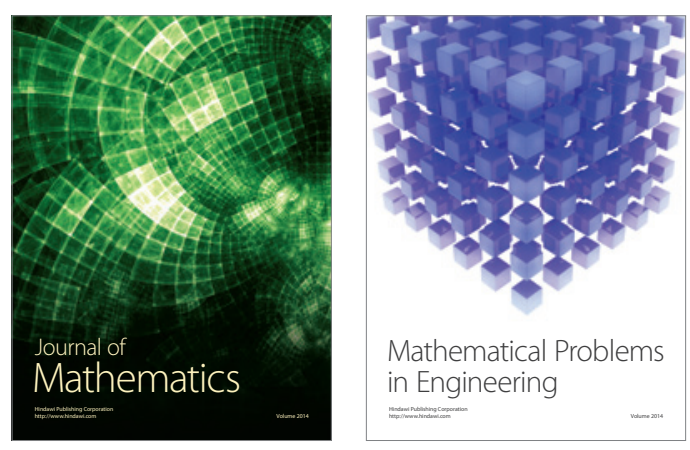

Mathematical Problems in Engineering
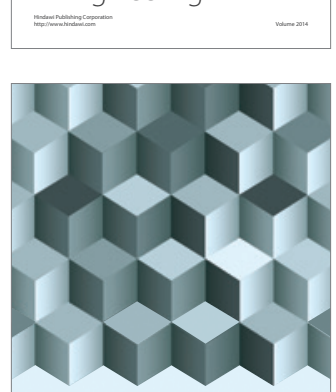

Journal of

Function Spaces
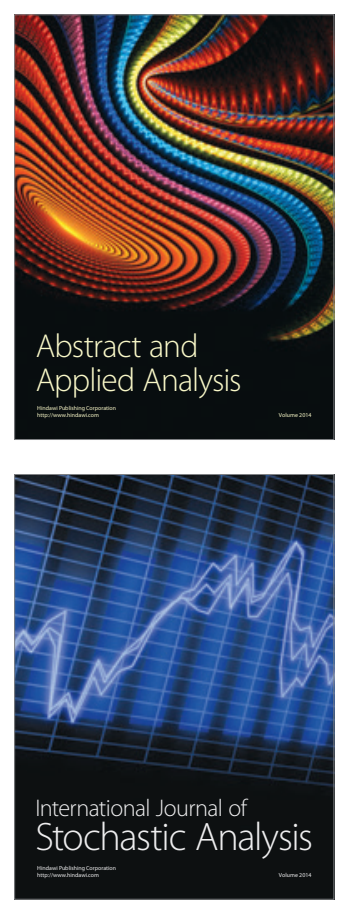

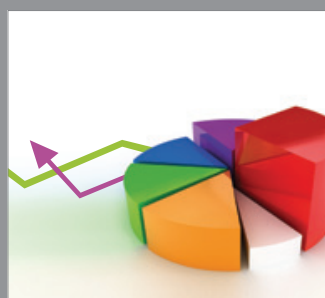

ournal of

Probability and Statistics

Promensencen
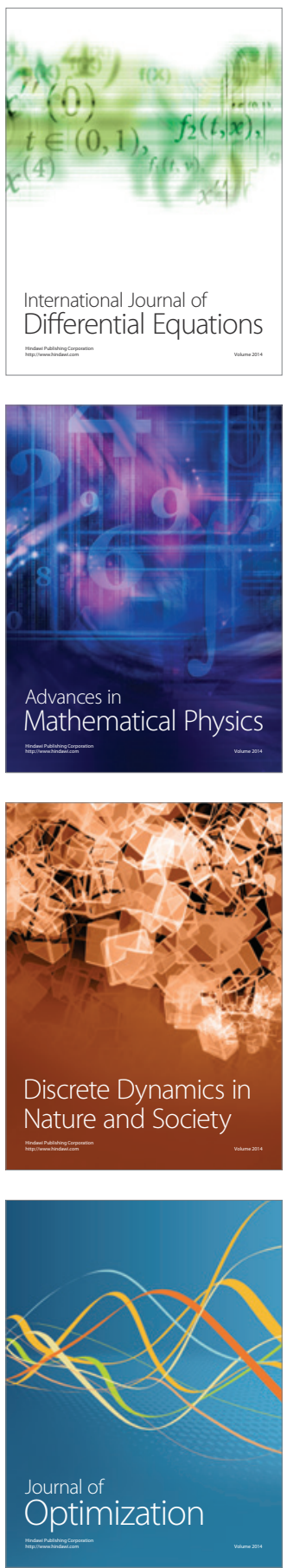